\title{
A Comparison Among Simple Algorithms for Linear Programming
}

\author{
J. SILVA $^{1 *}$, C.T.L.S. GHIDINI ${ }^{2}$, A.R.L. OLIVEIRA ${ }^{3}$ and M.I.V. FONTOVA ${ }^{4}$ \\ Received on July 28, 2017 / Accepted on March 10, 2018
}

\begin{abstract}
This paper presents a comparison between a family of simple algorithms for linear programming and the optimal pair adjustment algorithm. The optimal pair adjustment algorithm improvements the convergence of von Neumann's algorithm which is very attractive because of its simplicity. However, it is not practical to solve linear programming problems to optimality, since its convergence is slow. The family of simple algorithms results from the generalization of the optimal pair adjustment algorithm, including a parameter on the number of chosen columns instead of just a pair of them. Such generalization preserves the simple algorithms nice features. Significant improvements over the optimal pair adjustment algorithm were demonstrated through numerical experiments on a set of linear programming problems.
\end{abstract}

Keywords: Linear programming, von Neumann's algorithm, Simple algorithms.

\section{INTRODUCTION}

The von Neumann algorithm was reported by Dantzig in the early 1990s [4, 5], and it was later studied by Epelman and Freund [7], and Beck and Teboulle [1]. Some of the advantages presented by this method are its low computational cost per iteration, which is dominated by the matrix-vector multiplication, in addition to its ability to exploit the data sparsity from the original problem and the usually fast initial advance. Epelman and Freund [7] refer to this algorithm as "elementary", since each iteration involves only simple computations; therefore, it is unsophisticated, especially when compared with the modern interior point algorithms.

In [11], three algorithms were proposed to overcome some convergence difficulties from von Neumann's method: the optimal pair adjustment algorithm, the weight reduction algorithm,

\footnotetext{
*Corresponding author: Jair Silva - E-mail: jairsilva@ufpr.br

${ }^{1}$ Campus Avançado da Universidade Federal do Paraná em Jandaia do Sul, Rua Doutor João Maximiano, 426, Vila Operária, 86900-000, Jandaia do Sul, PR, Brazil. E-mail: jairsilva@ufpr.br

2 Faculdade de Ciências Aplicadas, Universidade de Campinas, Rua Pedro Zaccaria, 1300, 13484-350, Limeira, SP, Brazil. E-mail: carla.ghidini@fca.unicamp.br

${ }^{3}$ Instituto de Matemática, Estatística e Computação Científica, Universidade de Campinas, Rua Sérgio Buarque de Holanda, 651, 13083-859, Campinas, SP, Brazil. E-mail: aurelio@ime.unicamp.br

${ }^{4}$ Centro Universitário de Campo Limpo Paulista, Rua Guatemala, 167, 13231-230, Jd.América, Campo Limpo Paulista, SP, Brazil. E-mail: martavelazco@cc.faccamp.br
} 
and projection algorithm. The optimal pair adjustment algorithm (OPAA) provides the best results among them. This algorithm inherits the best properties from von Neumann's algorithm. Although OPAA has a faster convergence when compared to von Neumann's algorithm, its convergence is also considered slow, making it impractical for solving linear optimization problems.

This work presents a comparison between a family of simple algorithms for linear programming and the optimal pair adjustment algorithm. This family originated from the generalization of the idea presented by Gonçalves, Storer and Gondzio in [11] to develop the OPAA. Hence, the optimal adjustment algorithm for $p$ coordinates was developed. Indeed, for different values of $p$, a different algorithm is defined, in which $p$ is limited by the order of the problem, thus resulting in a family of algorithms. This family of simple algorithms maintains the ability to exploit the sparsity from the original problem and a fast initial convergence. Significant improvements over OPAA are demonstrated through numerical experiments on a set of linear programming problems.

The paper is organized as follows. Section 2 contains a description of von Neumann's algorithm. Section 3 presents both the weight reduction algorithm and the OPAA. Section 4 discusses the family of simple algorithms, theoretical properties of convergence of the optimal adjustment algorithm for $p$ coordinates, and a sufficient condition for it to present better iterations than the iterations of von Neumann's algorithm. Section 5 describes the computational experiments comparing the family with the OPAA. The conclusions and perspectives for future work are presented in the last section.

\section{THE VON NEUMANN'S ALGORITHM}

The von Neumann algorithm for solving linear programming problems was first described by Dantzig in the early 1990s in $[4,5]$. Such an algorithm actually solves the equivalent problem described below.

Consider the following set of linear constraints and the the search for a feasible solution for:

$$
\begin{aligned}
& P x=0 \\
& e^{t} x=1, x \geq 0
\end{aligned}
$$

where $P \in \mathfrak{R}^{m \times n}$ and $\left\|P_{j}\right\|=1$ for $j=1, \ldots, n$ (the columns have norm one), $x \in \mathfrak{R}^{n}, e \in \mathfrak{R}^{n}$ is the vector with all ones.

Geometrically, the columns $P_{j}$ are points on the $m$-dimensional hypersphere with unit radius and center at the origin. Therefore, the above problem assigns non-negative weights $x_{j}$ to the $P_{j}$ columns so that its origin is the rescaled gravity center. Note that any linear programming problem can be reduced to problem (2.1) (see [10]).

Figure 1 shows the algorithm. In the $k-t h$ iteration, the residual is $P x^{k}=b^{k-1}$. The next residual $b^{k}$ is the projection of the origin in the segment of line joining $b^{k-1}$ to $P_{s}$, where $P_{s}$ is the column 
that forms the largest angle with the residual $b^{k-1}$. The triangle $b^{k-1} 0 b^{k}$ has as hypotenuse $0 b^{k-1}$ and cathetus $0 b^{k}$, and thus, $\left\|b^{k}\right\|<\left\|b^{k-1}\right\|$ for all iterations.

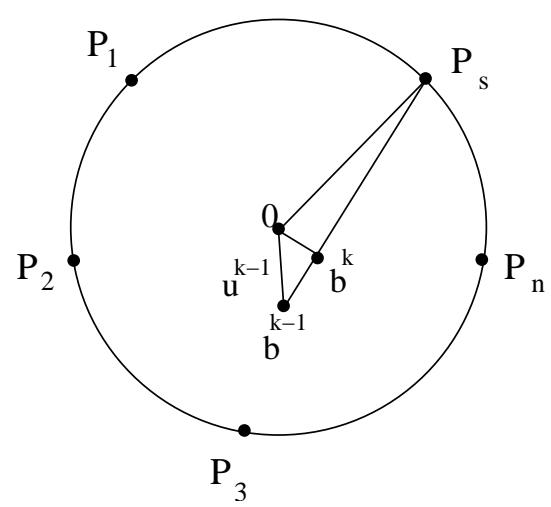

Figure 1: Illustration of von Neumann's algorithm.

The steps of von Neumann's algorithm are described below:

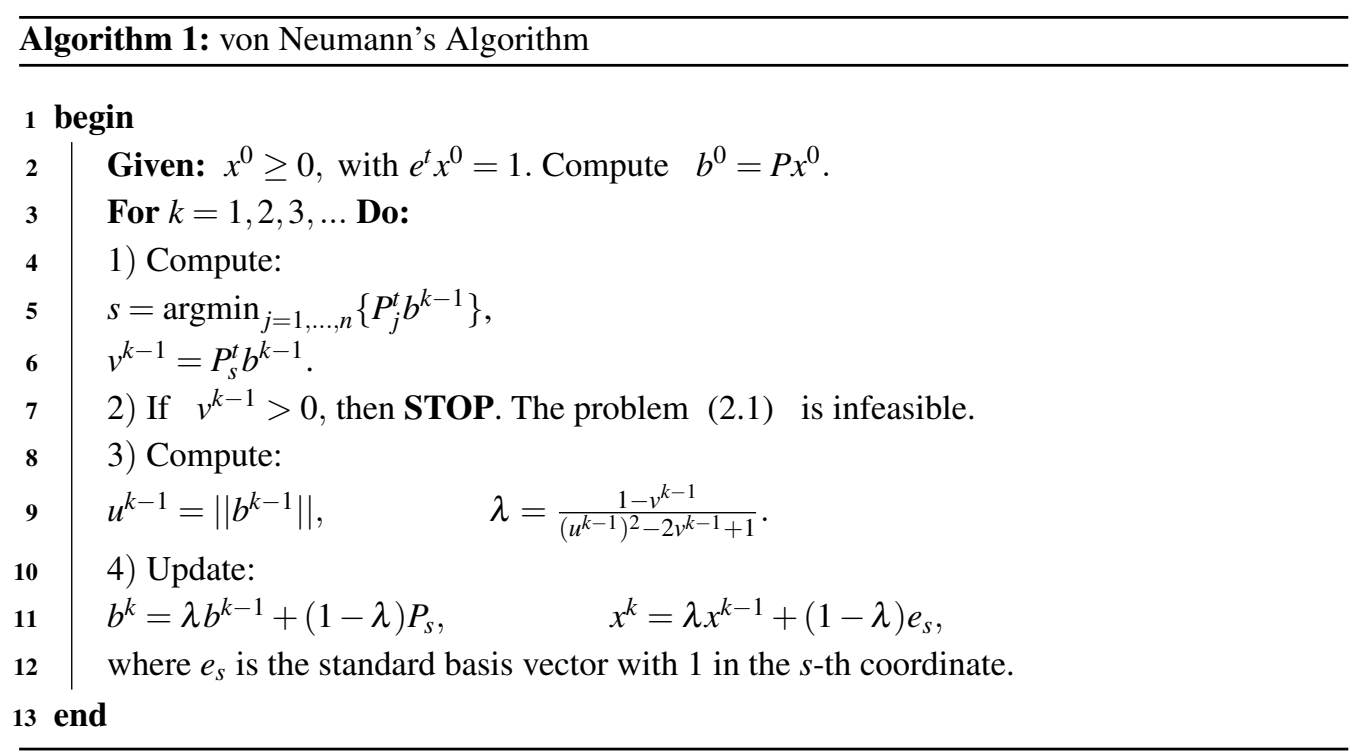

In computational experiments, the stopping criterion was $\left\|b^{k}-b^{k-1}\right\| /\left\|b^{k}\right\|<\varepsilon$, where $\varepsilon$ is a specified tolerance, and $x_{j}^{0}=\frac{1}{n}, j=1, \ldots, n$ was considered.

The effort per iteration of von Neumann's algorithm is dominated by the matrix-vector multiplication, required for the selection of the column $P_{s}$, which is $O(m n)$. The number of operations required in this multiplication is significantly lower, if the $P$ matrix is sparse. For more details, see $[10,11]$. 


\section{THE WEIGHT REDUCTION AND THE OPTIMAL PAIR ADJUSTMENT ALGORITHMS}

In this section, two algorithms developed by Gonçalves [11] are described. The algorithms are based on von Neumann's algorithms and were developed to improve convergence. They are the weight-reduction algorithm and the optimal pair adjustment algorithm.

In the weight-reduction algorithm, the residual $b^{k-1}$ is moved closer to the origin 0 by increasing the weight $x_{j}$ of some columns $P_{j}$ or decreasing the weight $x_{i}$ of other columns $P_{i}$. Figure 2 shows the geometric interpretation of the weight-reduction algorithm.

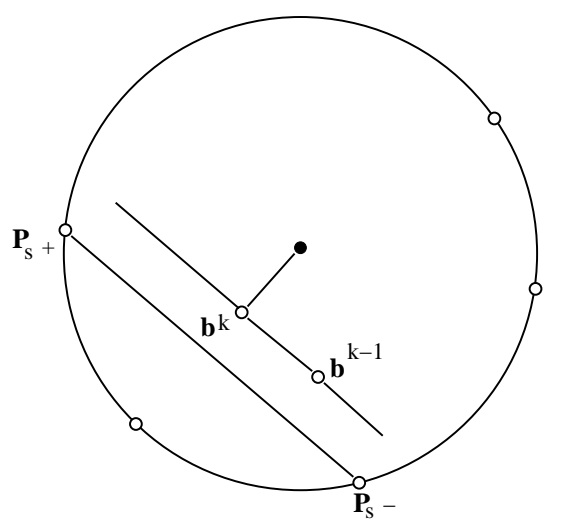

Figure 2: Illustration of the weight-reduction algorithm.

At each iteration, the residual $b^{k-1}$ moves in the direction $P_{s^{+}}-P_{s^{-}}$where the columns $P_{s^{+}}$ and $P_{s^{-}}$make the largest and smallest angle with $b^{k-1}$, respectively. The new residual $b^{k}$ is the projection of the origin in that line. Only the weights $x_{+}$and $x_{-}$will be changed. There is no guarantee that an iteration of this algorithm will improve as much as an iteration of the von Neumann algorithm [11].

However, the OPAA also developed by Gonçalves improves the residual at least as much as the von Neumann algorithm [11].

First, the OPAA calculates the columns $P_{s^{+}}$and $P_{s^{-}}$. Then, the algorithm computes the values $x_{s^{+}}^{k}, x_{s^{-}}^{k}$ and $\lambda$ where $x_{j}^{k}=\lambda x_{j}^{k-1}$ for all $j \neq s^{+}$and $j \neq s^{-}$that minimize the distance between $b^{k}$ and the origin, subject to the convexity and the non-negativity constraints. The solution to this optimization problem is easy to find by examining the Karush-Kuhn-Tucker (KKT) conditions (see [11]).

The optimal pair adjustment algorithm is described below.

The OPAA modifies all the weights $x_{i^{\prime} s}^{k}$, while the weight reduction algorithm modifies only the weights of columns $P_{s^{+}}$and $P_{s^{-}}$. This is the main difference between them. 


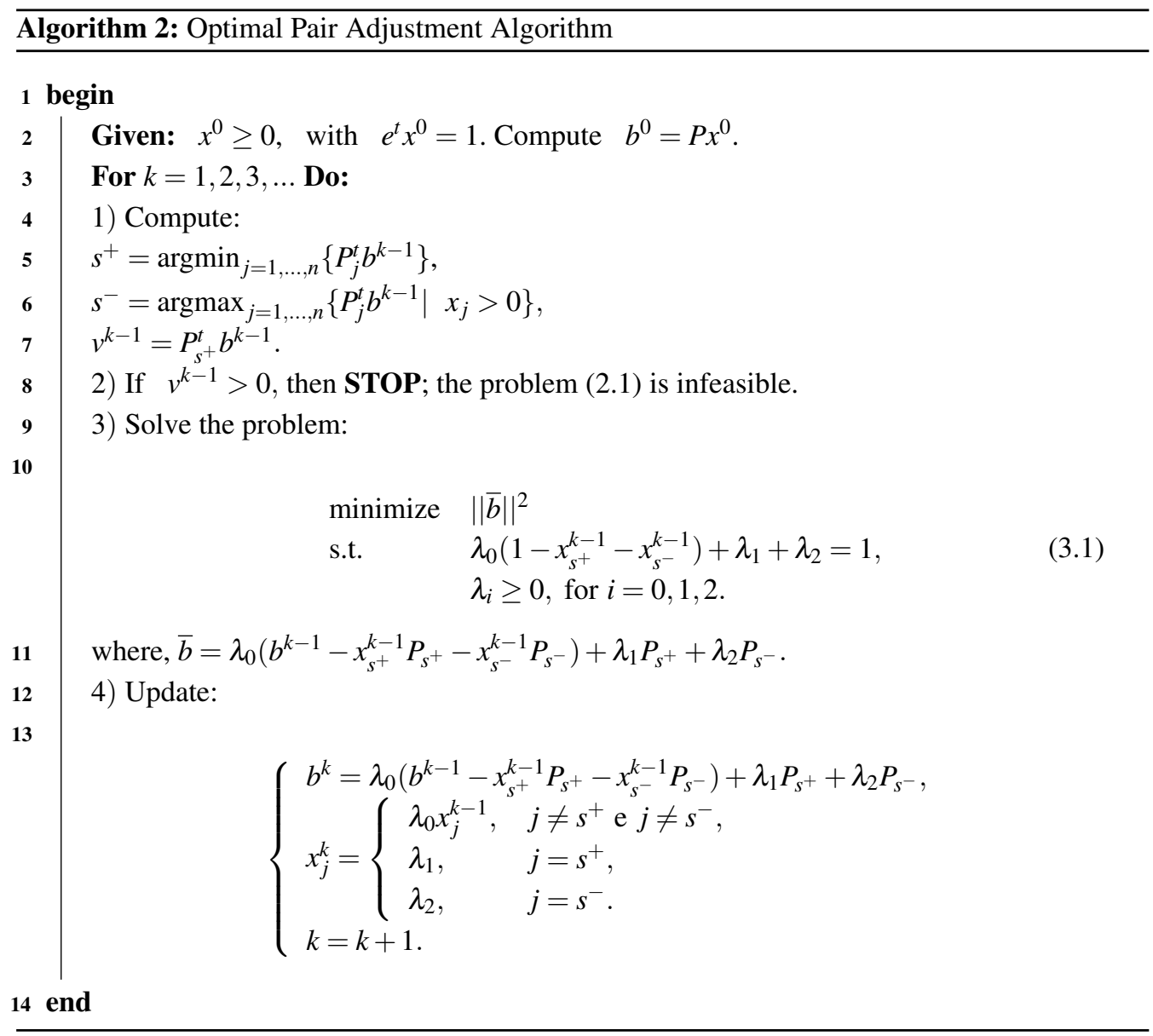

\section{OPTIMAL ADJUSTMENT ALGORITHM FOR $P$ COORDINATES}

This section presents the optimal adjustment algorithm for $p$ coordinates developed by Silva [12]. This algorithm was developed by generalizing the idea presented in the subproblem (10) of the OPAA. Instead of using two columns to formulate the subproblem, any number of columns can be used, thus assigning relevancy to any number of variables. For each value of $p$, a different algorithm can be formulated. Thus, a family of algorithms was developed.

The $p$ variables can be chosen by a different method according to the problem. A natural choice is to take $p / 2$ columns that make the largest angle with the vector $b^{k}$ and $p / 2$ columns that make the smallest angle with the vector $b^{k}$. If $p$ is an odd number, an extra column for the set of vectors is taken, which form the largest angle with the vector $b^{k}$, for instance.

The optimal adjustment algorithm for $p$ coordinates computes better direction than the OPAA. It still maintains simplicity, since at each iteration, only a matrix-vector multiplication is performed and a small linear system with a positive definite matrix is solved. 
The steps of the optimal adjustment algorithm for $p$ coordinates are similar to those for the OPAA. First, the $s_{1}$ and $s_{2}$ columns are identified; the $s_{1}$ and $s_{2}$ columns form the largest and the smallest angle with the residual $b^{k-1}$, respectively, where $s_{1}+s_{2}=p$ and $p$ is the number of columns to be prioritized. Next, the subproblem is solved, and then, the residual and the current point are updated.

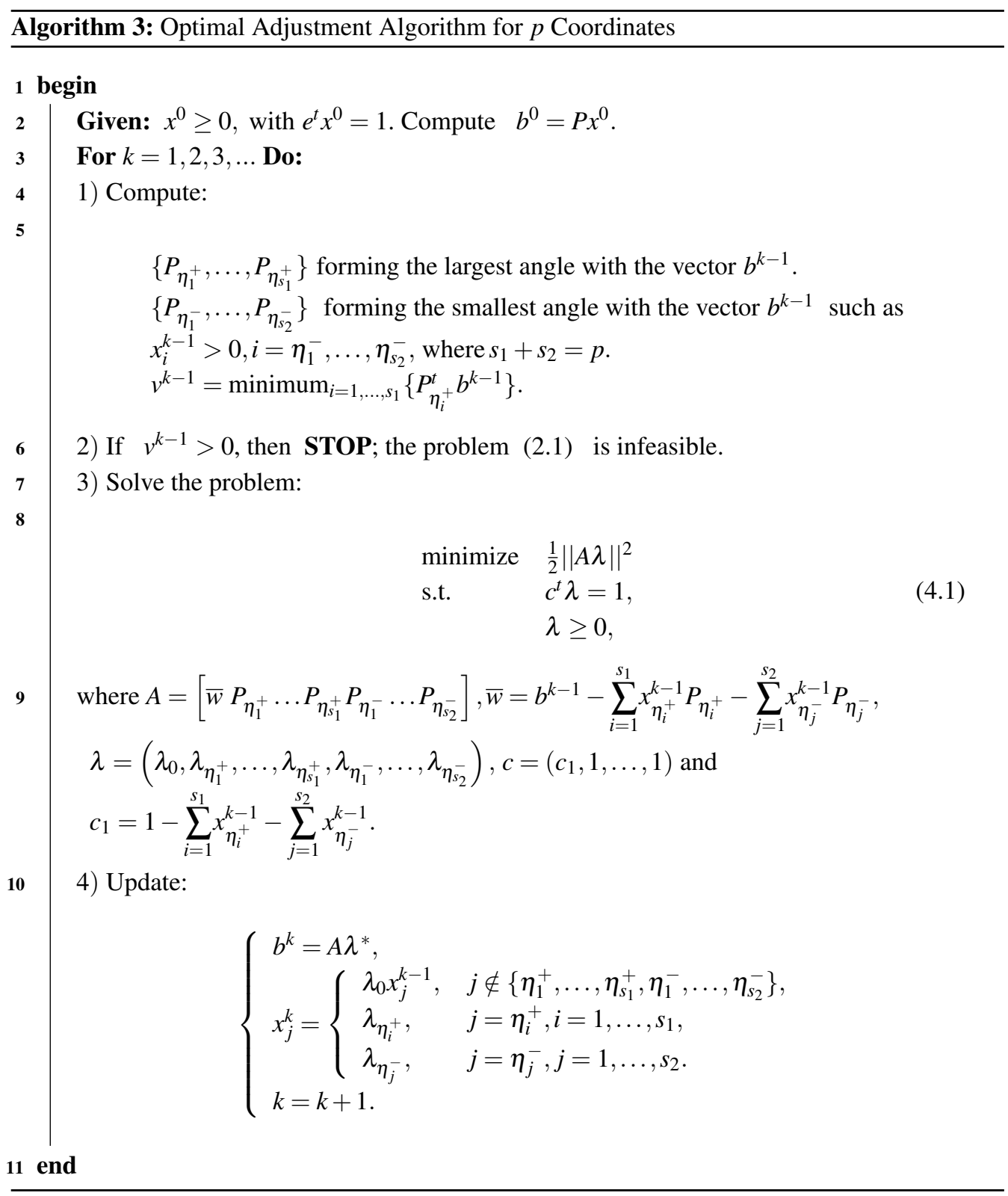


Subproblem (8) was built in such a way that the residual has the maximum decrease, such that, $x^{0} \geq 0$, with $e^{t} x^{0}=1$.

\subsection{Relation between von Neumann's algorithm and the algorithm with $p=1$ and geometric interpretation}

The optimal adjustment algorithms for $p$ coordinates with $p=1$ and von Neumann's algorithm generate the same points $x^{k}$ and the same residual $b^{k}$ as shown in Figure 1.

The optimal $\lambda$ for von Neumann's algorithm is calculated by the projection of the origin in the segment of line joining $b^{k-1}$ to $P_{s}$.

In the algorithm when $p=1$, the following subproblem is solved.

$$
\begin{array}{ll}
\operatorname{minimize} & \|\bar{b}\|^{2} \\
\text { s.t. } & \lambda_{0}\left(1-x_{s}^{k-1}\right)+\lambda_{1}=1, \\
& \lambda_{i} \geq 0, \text { for } i=0,1 .
\end{array}
$$

where, $\bar{b}=\lambda_{0}\left(b^{k-1}-x_{s}^{k-1} P_{s}\right)+\lambda_{1} P_{s}$.

The subproblem can be rewritten (4.2) as follows:

$$
\begin{aligned}
& \lambda_{0}\left(1-x_{s}^{k-1}\right)+\lambda_{1}=1 \Leftrightarrow \lambda_{1}=1-\lambda_{0}\left(1-x_{s}^{k-1}\right) \geq 0 \\
& \bar{b} \quad=\lambda_{0}\left(b^{k-1}-x_{s}^{k-1} P_{s}\right)+\lambda_{1} P_{s} \\
& =\lambda_{0}\left(b^{k-1}-x_{s}^{k-1} P_{s}\right)+\left(1-\lambda_{0}\left(1-x_{s}^{k-1}\right)\right) P_{s} \\
& =\lambda_{0} b^{k-1}+\left(1-\lambda_{0}\right) P_{s} .
\end{aligned}
$$

Thus, the problem (4.2) will be:

$$
\begin{array}{ll}
\operatorname{minimize} & \|\bar{b}\|^{2} \\
\text { s.t. } & \lambda \in\left[0, \frac{1}{1-x_{s}^{k-1}}\right]
\end{array}
$$

where, $\bar{b}=\lambda b^{k-1}+(1-\lambda) P_{s}$.

If the term $\frac{1}{1-x_{s}^{k-1}}$ is larger than 1 , then there will be an increase in the number of possible solutions in comparison with von Neumann's algorithm. Although, the geometric interpretation of the algorithm with $p=1$ presented in Figure 3 show that the optimal $\lambda$ is the same for both algorithms.

In Step 4 of von Neumann's algorithm, $x^{k}$ is updated by

$x_{j}^{k}= \begin{cases}\lambda x_{j}^{k-1}, & j \neq s \\ \lambda\left(x_{s}^{k-1}-1\right)+1, & j=s .\end{cases}$ 


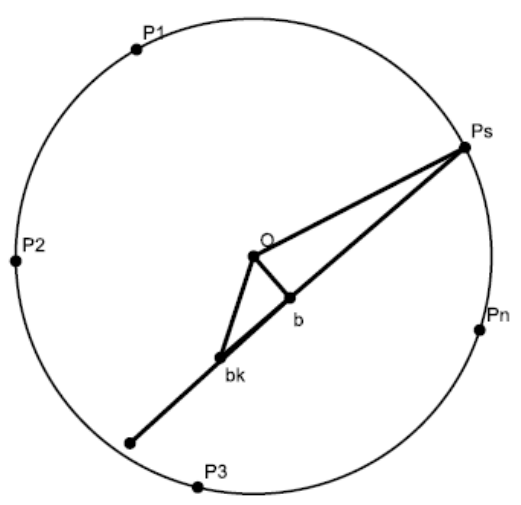

Figure 3: Illustration of the algorithm with $p=1$.

In Step 4 of the algorithm with $p=1, x^{k}$ is updated by

$x_{j}^{k}= \begin{cases}\lambda x_{j}^{k-1}, & j \neq s \\ 1-\lambda\left(1-x_{s}^{k-1}\right), & j=s .\end{cases}$

Therefore, the algorithm with $p=1$ and von Neumann's algorithm generate the same points $x^{k}$ and the same residual $b^{k}$.

For $p=2$, the subproblem (8) is reduced to the following form:

$$
\begin{array}{ll}
\operatorname{minimize} & \|\bar{b}\|^{2} \\
\text { s.t. } & \lambda_{0}\left(1-x_{s^{+}}^{k-1}-x_{s^{-}}^{k-1}\right)+\lambda_{1}+\lambda_{2}=1, \\
& \lambda_{i} \geq 0, \text { for } i=0,1,2 .
\end{array}
$$

where, $\bar{b}=\lambda_{0}\left(b^{k-1}-x_{s^{+}}^{k-1} P_{s^{+}}-x_{s^{-}}^{k-1} P_{s^{-}}\right)+\lambda_{1} P_{s^{+}}+\lambda_{2} P_{s^{-}}$.

The term $\bar{b}$ can be rewritten as:

$$
\begin{aligned}
\bar{b} & =\lambda_{0}\left(b^{k-1}-x_{s^{+}}^{k-1} P_{s^{+}}-x_{s^{-}}^{k-1} P_{s^{-}}\right)+\lambda_{1} P_{s^{+}}+\lambda_{2} P_{s^{-}} \\
& =\lambda_{0} b^{k-1}+\left(\lambda_{1}-\lambda_{0} x_{s^{+}}^{k-1}\right) P_{s^{+}}+\left(\lambda_{2}-\lambda_{0} x_{s^{-}}^{k-1}\right) P_{s^{-}}
\end{aligned}
$$

Thus, $\bar{b}\left(\lambda_{0}, \lambda_{1}, \lambda_{2}\right)$ is a linear transformation. When the vectors $\left\{\left(b^{k-1}-x_{s^{+}}^{k-1} P_{s^{+}}-x_{s^{-}}^{k-1} P_{s^{-}}\right), P_{s^{+}}\right.$ e $\left.P_{s^{-}}\right\}$are linearly independent, such linear transformation is injective. It transforms the triangle generated by $\lambda_{0}\left(1-x_{s^{+}}^{k-1}-x_{s^{-}}^{k-1}\right)+\lambda_{1}+\lambda_{2}=1$ and its interior into the triangle whose vertices are $P_{s^{+}}, P_{s^{-}}$and $P_{v}=\frac{1}{\left(1-x_{s^{+}}^{k-1}-x_{s^{-}}^{k-1}\right)}\left(b^{k-1}-x_{s^{+}}^{k-1} P_{s^{+}}-x_{s^{-}}^{k-1} P_{s^{-}}\right)$, and its interior.

Therefore, the optimal residual $b^{k}$ is the projection of the origin on this triangle. The geometric interpretation of the algorithm with $p=2$ is given in Figure 4.

For $p>2$ coordinates, the subproblem (8) will be 


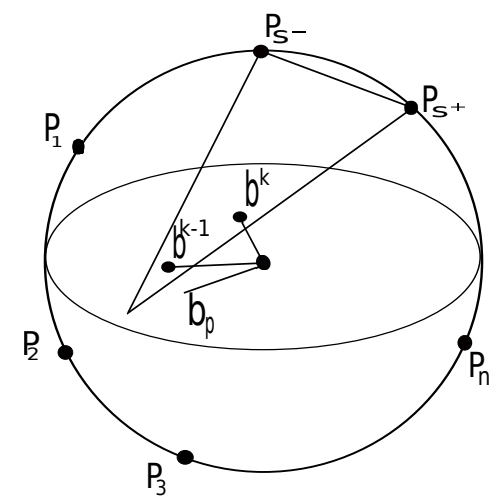

Figure 4: Illustration of the algorithm with $p=2$.

$$
\begin{aligned}
\bar{b} & =\lambda_{0}\left(b^{k-1}-\sum_{i=1}^{s_{1}} x_{\eta_{i}^{+}}^{k-1} P_{\eta_{i}^{+}}-\sum_{j=1}^{s_{2}} x_{\eta_{j}^{-}}^{k-1} P_{\eta_{j}^{-}}\right)+\sum_{i=1}^{s_{1}} \lambda_{\eta_{i}^{+}} P_{\eta_{i}^{+}}+\sum_{j=1}^{s_{2}} \lambda_{\eta_{j}^{-}} P_{\eta_{j}^{-}} \\
& =\lambda_{0} b^{k-1}+\sum_{i=1}^{s_{1}}\left(\lambda_{\eta_{i}^{+}}-\lambda_{0} x_{\eta_{i}^{k}}^{k-1}\right) P_{\eta_{i}^{+}}+\sum_{j=1}^{s_{2}}\left(\lambda_{\eta_{j}^{-}}-\lambda_{0} x_{\eta_{j}^{k}}^{k-1}\right) P_{\eta_{j}^{-}}
\end{aligned}
$$

with $\lambda_{0}+\sum_{i=1}^{s_{1}}\left(\lambda_{\eta_{i}^{+}}-\lambda_{0} x_{\eta_{i}^{+}}^{k-1}\right)+\sum_{j=1}^{s_{2}}\left(\lambda_{\eta_{j}^{-}}-\lambda_{0} x_{\eta_{j}^{-}}^{k-1}\right)=1$, then $\bar{b}$ is also an affine combination. Then, the optimal residual $b^{k}$ is the projection of the origin on the affine space region with vertices in the $p$ columns and in vector $P_{v}$, and

$$
P_{v}=\frac{1}{1-\sum_{i=1}^{s_{1}} x_{\eta_{i}^{+}}^{k-1}-\sum_{j=1}^{s_{2}} x_{\eta_{j}^{k-1}}^{k}}\left(b^{k-1}-\sum_{i=1}^{s_{1}} x_{\eta_{i}^{+}}^{k-1} P_{\eta_{i}^{+}}-\sum_{j=1}^{s_{2}} x_{\eta_{j}^{-}}^{k-1} P_{\eta_{j}^{-}}\right)
$$

\subsection{Subproblem Solution Using Interior Point Methods}

In [11], Gonçalves solved the subproblem (10) by checking all feasible solutions that satisfies the KKT condition of this subproblem and there are $2^{3}-1$ possible solutions, see [11]. For the optimal adjustment algorithm for $p$ Coordinates, the possible solutions of the subproblem (8) are $2^{p}-1$ [9]. The strategy used by Gonçalves in [11] is inefficient even for small values of $p$; the number of cases will increase exponentially. The subproblem (8) can be solved by interior point methods.

Consider the subproblem (8). The KKT equations from the problem (8) are given by:

$$
\begin{aligned}
A^{t} A \lambda+c \tau-\mu & =0, \\
\mu^{t} \lambda & =0, \\
c^{t} \lambda-1 & =0, \\
-\lambda & \leq 0,
\end{aligned}
$$


where $\tau$ is a vector of free variables and $0 \leq \mu$. The vectors $\tau$ and $\mu$ are the Lagrange multipliers for equality and inequality constraints, respectively, and $A^{t} A$ is a $(p+1) \times(p+1)$ matrix.

The path-following interior point method is used to solve the problem (4.6). At each iteration of the interior point method, the linear system of Equation (4.7) is solved to compute the search directions $(d \lambda, d \tau, d \mu)$ :

$$
\left[\begin{array}{ccc}
A^{t} A & c & -I d \\
U & 0 & \Lambda \\
c^{t} & 0 & 0
\end{array}\right]\left[\begin{array}{c}
d \lambda \\
d \tau \\
d \mu
\end{array}\right]=\left[\begin{array}{l}
r_{1} \\
r_{2} \\
r_{3}
\end{array}\right]
$$

where $U=\operatorname{diag}(\mu), \Lambda=\operatorname{diag}(\lambda), r_{1}=\mu-c \tau-A^{t} A \lambda, r_{2}=-\tau^{t} \lambda$, and $r_{3}=1-c^{t} \lambda$.

The directions $d \lambda, d \tau$ and $d \mu$ are given by:

$$
\begin{aligned}
& d \mu=\Lambda^{-1} r_{2}-\Lambda^{-1} U d \lambda, \\
& d \lambda=\left(A^{t} A+\Lambda^{-1} U\right)^{-1} r_{4}-\left(A^{t} A+\Lambda^{-1} U\right)^{-1} c d \tau, \\
& c^{t}\left(A^{t} A+\Lambda^{-1} U\right)^{-1} c d \tau=c^{t}\left(A^{t} A+\Lambda^{-1} U\right)^{-1} r_{4}-r_{3},
\end{aligned}
$$

where $r_{4}=r_{1}+\Lambda^{-1} r_{2}$.

Consider $l_{1}=\left(A^{t} A+\Lambda^{-1} U\right)^{-1} c$ and $l_{2}=\left(A^{t} A+\Lambda^{-1} U\right)^{-1} r_{4}$; then, the solution of the linear systems $\left(A^{t} A+\Lambda^{-1} U\right) l_{1}=c$ and $\left(A^{t} A+\Lambda^{-1} U\right) l_{2}=r_{4}$ will be necessary to compute the directions.

The matrix $A^{t} A+\Lambda^{-1} U$ is a symmetric $(p+1) \times(p+1)$ positive definite. Both systems can be solved with the same Cholesky factorization.

\subsection{Theoretical Properties of the Family of Algorithms}

The theorem 3.1 in [11] ensures that the OPAA converges, in the worst case, with the same rate of convergence as von Neumann's algorithm. This result can be extended for the optimal adjustment algorithm for $p$ coordinates, and an increase in the value of $p$ leads to a more efficient algorithm with improved performance. This is shown in Theorem 4.1. Only the second part will be proved.

Teorema 4.1. The residual $\| b^{k}||$ after an iteration of the optimal adjustment algorithm for $p$ coordinates is, in the worst case, equal to the residual after an iteration of von Neumann's algorithm. Furthermore, suppose that $\left\|b_{p_{1}}^{k}\right\|$ is the residual after an iteration of the optimal adjustment algorithm for $p_{1}$ coordinates, $\left\|b_{p_{2}}^{k}\right\|$ is the residual after an iteration of the optimal adjustment algorithm for $p_{2}$ coordinates, and $p_{1} \leq p_{2} \leq n$, then $\left\|b_{p_{1}}^{k}\right\| \leq\left\|b_{p_{2}}^{k}\right\|$ where $n$ is the number of columns $P$.

Proof. Let $k \geq 1$ and $b^{k-1}$ be the residual at the beginning of the iteration $k$. Further, $\left\{P_{\eta_{1}^{+}}, \ldots, P_{\eta_{s_{1}}^{+}}\right\}$and $\left\{P_{\eta_{1}^{-}}, \ldots, P_{\eta_{s_{2}}^{-}}\right\}$are sets of vectors forming the largest and the smallest angles with the vector $b^{k-1}$, respectively, for the algorithm prioritizing the $p_{2}$ coordinates, where 
$s_{1}+s_{2}=p_{2}$; and $\left\{P_{\eta_{1}^{+}}, \ldots, P_{\eta_{s_{3}^{+}}}\right\}$and $\left\{P_{\eta_{1}^{-}}, \ldots, P_{\eta_{s_{4}}^{-}}\right\}$are sets of vectors forming the largest and the smallest angles with the vector $b^{k-1}$, respectively, for the algorithm prioritizing the $p_{1}$ coordinates, where $s_{3}+s_{4}=p_{1}$.

After the $k$-th iteration in the optimal adjustment for the $p_{2}$ coordinates, the residual $b_{p_{2}}^{k}$ will be

$$
b_{p_{2}}^{k}=\bar{\lambda}_{1}\left(b^{k-1}-\sum_{i=1}^{s_{1}} x_{\eta_{i}^{+}}^{k-1} P_{\eta_{i}^{+}}-\sum_{j=1}^{s_{2}} x_{\eta_{j}^{-}}^{k-1} P_{\eta_{j}^{-}}\right)+\sum_{i=1}^{s_{1}} \bar{\lambda}_{\eta_{i}^{+}} P_{\eta_{i}^{+}}+\sum_{j=1}^{s_{2}} \bar{\lambda}_{\eta_{j}^{-}} P_{\eta_{j}^{-}},
$$

where $\left(\bar{\lambda}_{1}, \bar{\lambda}_{\eta_{1}^{+}}, \ldots, \bar{\lambda}_{\eta_{s_{1}}^{+}}, \bar{\lambda}_{\eta_{1}^{-}}, \ldots, \bar{\lambda}_{\eta_{s_{2}}^{-}}\right)$is the optimal solution of the subproblem (8) prioritizing $p_{2}$ coordinates.

The optimal solution of the subproblem (8) prioritizing $p_{1}$ coordinates

$$
\left(\widetilde{\lambda}_{1}, \tilde{\lambda}_{\eta_{1}^{+}}, \ldots, \tilde{\lambda}_{\eta_{s_{3}}^{+}}, \widetilde{\lambda}_{\eta_{1}^{-}}, \ldots, \widetilde{\lambda}_{\eta_{s_{4}}^{-}}\right)
$$

is also a feasible solution for the subproblem (8) when $p_{2}$ coordinates are prioritized.

Therefore,

$$
\begin{aligned}
& \left\|\tilde{\lambda}_{1}\left(b^{k-1}-\sum_{i=1}^{s_{3}} x_{\eta_{i}^{+}}^{k-1} P_{\eta_{i}^{+}}-\sum_{j=1}^{s_{4}} x_{\eta_{j}^{-}}^{k-1} P_{\eta_{j}^{-}}\right)+\sum_{i=1}^{s_{3}} \tilde{\lambda}_{\eta_{i}^{+}} P_{\eta_{i}^{+}}+\sum_{j=1}^{s_{4}} \tilde{\lambda}_{\eta_{j}^{-}} P_{\eta_{j}^{-}}\right\|= \\
& =\left\|b_{p_{1}}^{k}\right\| \geq\left\|b_{p_{2}}^{k}\right\|,
\end{aligned}
$$

where $b_{p_{1}}^{k}$ is the residual after an iteration of the optimal adjustment algorithm for the $p_{1}$ coordinates. Consequently, the reduction of the residual after an iteration of the optimal adjustment algorithm for the $p_{2}$ coordinates is, in the worst case, equal to the reduction of the residual after an iteration of the optimal adjustment algorithm for the $p_{1}$ coordinates.

This theorem does not ensure that one iteration of family of algorithms is better than one iteration of von Neumann's algorithm. In the next section, we give the sufficient conditions for that to happen.

\subsection{Sufficient Conditions for $\left\|\boldsymbol{b}^{k}\right\|<\left\|\boldsymbol{b}_{v}^{k}\right\|$}

Let $b^{k}$ be the residual of the algorithm with $p=2$ in the iteration $k$, and let $P_{s^{+}}$and $P_{s^{-}}$be the columns forming the largest and smallest angles with the vector $b^{k-1}$. If the projection of the origin is in the interior of the triangle $b^{k} P_{s^{+}} P_{s^{-}}$and coincides with the projection of the origin in the plane determined by $b^{k-1}, P_{s^{+}}$and $P_{s^{-}}$, then $\left\|b^{k}\right\|<\left\|b_{v}^{k}\right\|$, where $b_{v}^{k}$ is the residual of von Neumann's algorithm in the iteration $k$. In fact, we can see this clearly in Figure 5, noting that the triangle $0 b^{k} b_{v}^{k}$ has the $\overline{0 b}_{v}^{k}$ hypotenuse and side $\overline{0 b}^{k}$.

Thus, we concluded that under these conditions, the OPAA for $p$ coordinates has better performance than von Neumann's algorithm. 


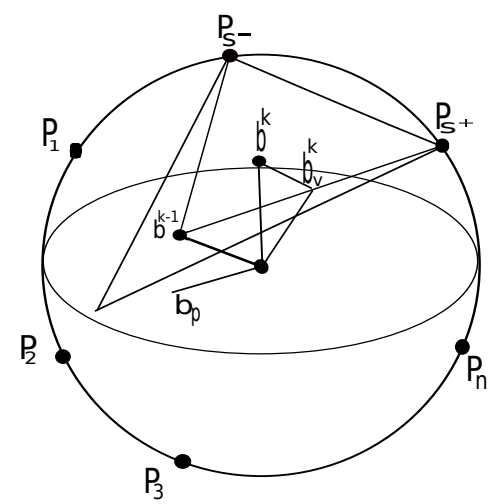

Figure 5: Illustration of the Sufficient Condition.

\section{COMPUTATIONAL EXPERIMENTS}

The algorithm with $\mathrm{p}=2$ is the same of the optimal pair adjustment algorithm. In particular, for the algorithm with $\mathrm{p}=2$, we could use the same strategy and compute all possible solutions of the subproblem, since there are only $2^{3}-1$ possible solutions. However, this strategy becomes impractical for larger values of $p$, so, the strategy is to solve the subproblem by interior point methods for all differents value of $\mathrm{p}$.

We make two experiments. In the first one, the performance of the family of algorithms for moderate values of $p$ is compared with OPAA, when $p=2$. The choice for moderate $p$ values comes from the fact that for larger $p$ values, the cost of solution of the subproblem in each iteration becomes noticeable.

The second experiment explores the fact that the optimal adjustment algorithm for $p$ coordinates allows a dynamic choice of $p$ considering the size of linear problem to be solved. This approach was proposed in [8], the developed heuristic was based on numerical experiments and its given by

\begin{tabular}{c}
$0<(\mathrm{m}+\mathrm{n}) \leq 10000 \rightarrow \mathrm{p}=4$ \\
$10000<(\mathrm{m}+\mathrm{n}) \leq 20000 \rightarrow \mathrm{p}=8$ \\
$20000<(\mathrm{m}+\mathrm{n}) \leq 400000 \rightarrow \mathrm{p}=20$ \\
$400000<(\mathrm{m}+\mathrm{n}) \leq 600000 \rightarrow \mathrm{p}=40$ \\
$600000<(\mathrm{m}+\mathrm{n}) \leq$ \\
\hline
\end{tabular}

where $m$ is the number of rows and $n$ is the number of columns for the constraint matrix $A$ of the linear problem.

For the second experiment, we proposed a new heuristic $p=\frac{n z(A)}{\sqrt{m n}}$ where $n z(A)$ is the number of nonzeros entries of $A$. And we compare this new heuristic with the heuristic proposed in [8]. 
For the all experiments, a collection of 151 linear programming problems is used. The problems are divided into 95 Netlib problems [3], 16 Kennington problems [2], and 40 other problems supplied by Gonçalves [11]. These experiments were performed on an Intel Core 2 Quad Q9550 $2.83 \mathrm{GHz}$ and 4GB of RAM machine in a Linux using the gec compiler.

\subsection{Implementation Details}

The family of algorithms is implemented in $\mathrm{C}$ using the format of the problem given in [10] Subsection 2.5.1. The matrix $P$ is not built explicitly. More precisely, it is divided into two blocks. Only one of these blocks is considered in Step 1, at each iteration, to find $s_{1}$ and $s_{2}$ columns, which form the largest and smallest angles with residual $b^{k}$, respectively.

To solve the subproblem, the classical path-following interior point method was implemented in C. The perturbation in the complementarity is $\frac{\mu^{t} \lambda}{(p+1)^{2}}$.

The initial point was the point with all coordinates equal to one. The tolerance is $10^{-12}$; since the linear equation $e^{t} x=1$ of the problem (2.1) makes each component $x_{j}$ small, if a solution with a good precision is not computed, the method may not work properly.

\subsection{Experiment Design}

The first experiment was performed following the steps presented by Gonçalves, Storer and Gondzio in [11]:

1. Initially, von Neumann's algorithm is run on all problems;

2. Next, when the relative difference between $\left\|b^{k-1}\right\|$ and $\left\|b^{k}\right\|$ was less than $0.5 \%$, the time $t 1$ (CPU seconds) and number of iterations (up to $t 1$ ) are recorded.

3. Additionally, the times $t 2, t 3, t 4$, and $t 5$ (CPU seconds), which correspond respectively to $3,5,10$ and 20 times the number of iterations in $t 1$ are also recorded.

4. Next, the optimal adjustment algorithm for $p$ coordinates, where $p=2, p=4, p=10$, $p=20, p=40$ and $p=100$ is ran on the test problems.

5. Finally, for the $t i$ times, $i=1, \ldots, 5$, the residual $\left\|b^{k}\right\|$ is recorded.

In the second experiment, both approaches use the termination criteria described in [8]. This is, the experiment stops when the algorithm exceeds its allotted maximum number of iterations (100) or when the relative error of the residual norm is smaller than a tolerance $10^{-4}$. The most successful approach is the one with the smaller residual $\left\|b^{k}\right\|$.

Table 1 shows the problems and the results for time $t 5$, which was used in the performance profile. We use time $t 5$, because in $t 5$ the algorithms get more time running. 
Table 1: Problems test and time t5

\begin{tabular}{|c|c|c|c|c|c|c|c|}
\hline Problem & Line & Column & Time $\mathbf{t 5}$ & Problem & Line & Column & Time $\mathbf{t 5}$ \\
\hline $25 \mathrm{fv} 47$ & 769 & 1821 & 0.060000 & ship041 & 292 & 1905 & 0.030000 \\
\hline $80 \mathrm{bau} 3 \mathrm{~b}$ & 1965 & 10701 & 0.240000 & ship04s & 216 & 1281 & 0.090000 \\
\hline adlittle & 53 & 134 & 0.000001 & ship081 & 470 & 3121 & 0.120000 \\
\hline afiro & 25 & 48 & 0.000001 & ship08s & 276 & 1604 & 0.100000 \\
\hline agg & 319 & 404 & 0.000001 & ship121 & 610 & 4171 & 0.040000 \\
\hline agg2 & 455 & 689 & 0.010000 & ship12s & 340 & 1943 & 0.150000 \\
\hline agg3 & 455 & 689 & 0.020000 & sierra & 1129 & 2618 & 0.080000 \\
\hline bandm & 211 & 366 & 0.000001 & stair & 356 & 531 & 0.060000 \\
\hline beaconfd & 73 & 148 & 0.020000 & standata & 292 & 582 & 0.050000 \\
\hline blend & 66 & 101 & 0.020000 & standgub & 292 & 582 & 0.050000 \\
\hline bnl1 & 558 & 1439 & 0.020000 & standmps & 388 & 1146 & 0.040000 \\
\hline bnl2 & 1848 & 3800 & 0.080000 & stocfor1 & 94 & 142 & 0.010000 \\
\hline boeing1 & 294 & 660 & 0.040000 & stocfor2 & 1968 & 2856 & 0.030000 \\
\hline boeing 2 & 125 & 264 & 0.000001 & stocfor3 & 15336 & 22202 & 0.190000 \\
\hline bore $3 \mathrm{~d}$ & 64 & 90 & 0.010000 & truss & 1000 & 8806 & 0.050000 \\
\hline brandy & 116 & 216 & 0.020000 & tuff & 246 & 553 & 0.120000 \\
\hline capri & 235 & 421 & 0.010000 & vtp_base & 46 & 82 & 0.000001 \\
\hline cycle & 1400 & 2749 & 0.020000 & wood1p & 171 & 1718 & 0.180000 \\
\hline czprob & 661 & 2705 & 0.040000 & woodw & 708 & 5364 & 0.090000 \\
\hline $\mathrm{d} 2 \mathrm{q} 06 \mathrm{c}$ & 2012 & 5561 & 0.130000 & cre-a & 2994 & 6692 & 0.040000 \\
\hline d6cube & 403 & 5443 & 0.480000 & cre-b & 5336 & 36382 & 0.230000 \\
\hline degen 2 & 444 & 757 & 0.050000 & cre-c & 2375 & 5412 & 0.030000 \\
\hline degen 3 & 1503 & 2604 & 0.260000 & cre-d & 4102 & 28601 & 0.190000 \\
\hline dfl001 & 5907 & 12065 & 1.440000 & ken-07 & 1427 & 2603 & 0.030000 \\
\hline e226 & 161 & 392 & 0.020000 & ken-11 & 10061 & 16709 & 0.540000 \\
\hline etamacro & 331 & 666 & 0.020000 & ken-13 & 22519 & 36546 & 2.060000 \\
\hline fffff800 & 313 & 817 & 0.040000 & ken-18 & 78823 & 128395 & 21.310000 \\
\hline finnis & 359 & 775 & 0.010000 & osa-07 & 1047 & 24911 & 0.160000 \\
\hline fit1d & 24 & 1047 & 0.010000 & osa-14 & 2266 & 54535 & 0.380000 \\
\hline fitlp & 678 & 1706 & 0.010000 & osa-30 & 4279 & 103978 & 0.000001 \\
\hline fit $2 d$ & 25 & 10387 & 0.280000 & osa-60 & 10209 & 242411 & 1.840000 \\
\hline fit $2 p$ & 3170 & 13695 & 0.240000 & pds- 02 & 2603 & 7333 & 0.070000 \\
\hline forplan & 104 & 411 & 0.090000 & pds-06 & 9119 & 28435 & 0.490000 \\
\hline ganges & 840 & 1197 & 0.030000 & pds- 10 & 15587 & 48719 & 1.240000 \\
\hline gfrd-pnc & 590 & 1134 & 0.020000 & pds-20 & 32287 & 106080 & 5.440000 \\
\hline greenbea & 1872 & 4081 & 0.070000 & $\mathrm{BL}$ & 5468 & 12038 & 0.830000 \\
\hline greenbeb & 1865 & 4065 & 0.090000 & BL2 & 5480 & 12063 & 0.840000 \\
\hline grow15 & 300 & 645 & 0.010000 & $\mathrm{CO} 5$ & 4471 & 10318 & 0.240000 \\
\hline grow 22 & 440 & 946 & 0.020000 & CO9 & 8510 & 19276 & 0.470000 \\
\hline
\end{tabular}


Table 1 (Continued from previous page)

\begin{tabular}{|c|c|c|c|c|c|c|c|}
\hline Problem & Line & Column & Time t5 & Problem & Line & Column & Time t5 \\
\hline grow7 & 140 & 301 & 0.000001 & CQ9 & 7073 & 17806 & 0.300000 \\
\hline israel & 166 & 307 & 0.010000 & GE & 8361 & 14096 & 0.200000 \\
\hline kb2 & 43 & 68 & 0.000001 & NL & 6478 & 14393 & 0.560000 \\
\hline lotfi & 117 & 329 & 0.000001 & a1 & 42 & 73 & 0.000001 \\
\hline maros & 626 & 1365 & 0.030000 & fort45 & 1037 & 1467 & 0.060000 \\
\hline maros-r7 & 2152 & 6578 & 0.090000 & fort46 & 1037 & 1467 & 0.060000 \\
\hline modszk1 & 658 & 1405 & 0.010000 & fort47 & 1037 & 1467 & 0.050000 \\
\hline nesm & 646 & 2850 & 0.080000 & fort48 & 1037 & 1467 & 0.060000 \\
\hline perold & 580 & 1412 & 0.020000 & scagr 25 & 344 & 543 & 0.020000 \\
\hline pilot & 1350 & 4506 & 0.090000 & fort49 & 1037 & 1467 & 0.050000 \\
\hline pilot4 & 389 & 1069 & 0.030000 & fort51 & 1042 & 1473 & 0.060000 \\
\hline pilot87 & 1968 & 6367 & 0.220000 & fort52 & 1041 & 1471 & 0.060000 \\
\hline pilot_ja & 795 & 1834 & 0.040000 & fort53 & 1041 & 1471 & 0.050000 \\
\hline pilot_we & 691 & 2621 & 0.050000 & fort54 & 1041 & 1471 & 0.050000 \\
\hline pilotnov & 830 & 2089 & 0.050000 & fort55 & 1041 & 1471 & 0.060000 \\
\hline recipe & 61 & 120 & 0.000001 & fort56 & 1041 & 1471 & 0.040000 \\
\hline sc105 & 104 & 162 & 0.000001 & fort57 & 1041 & 1471 & 0.060000 \\
\hline $\operatorname{sc} 205$ & 203 & 315 & 0.000001 & fort58 & 1041 & 1471 & 0.050000 \\
\hline $\mathrm{sc} 50 \mathrm{a}$ & 49 & 77 & 0.000001 & fort59 & 1041 & 1471 & 0.050000 \\
\hline $\mathrm{sc} 50 \mathrm{~b}$ & 48 & 76 & 0.000001 & fort60 & 1041 & 1471 & 0.060000 \\
\hline scagr 25 & 344 & 543 & 0.010000 & fort61 & 1041 & 1471 & 0.050000 \\
\hline scagr7 & 92 & 147 & 0.000001 & $\mathrm{x} 1$ & 983 & 1413 & 0.050000 \\
\hline scfxm1 & 268 & 526 & 0.020000 & $\mathrm{x} 2$ & 983 & 1413 & 0.050000 \\
\hline $\operatorname{scfxm} 2$ & 536 & 1052 & 0.030000 & pata01 & 122 & 1241 & 0.010000 \\
\hline $\operatorname{scfxm} 3$ & 804 & 1578 & 0.040000 & pata02 & 122 & 1241 & 0.020000 \\
\hline scorpion & 180 & 239 & 0.060000 & patb01 & 57 & 143 & 0.000001 \\
\hline scrs8 & 418 & 1183 & 0.080000 & patb02 & 57 & 143 & 0.000001 \\
\hline $\operatorname{scsd} 1$ & 77 & 760 & 0.260000 & vschna02 & 122 & 1363 & 0.010000 \\
\hline $\operatorname{scsd} 6$ & 148 & 1350 & 0.430000 & vschnb01 & 57 & 144 & 0.000001 \\
\hline $\operatorname{scsd} 8$ & 397 & 2750 & 0.070000 & vschnb02 & 58 & 202 & 0.000001 \\
\hline sctap1 & 269 & 608 & 0.010000 & willett & 184 & 588 & 0.030000 \\
\hline sctap2 & 977 & 2303 & 0.010000 & ex01 & 234 & 1555 & 0.070000 \\
\hline sctap3 & 1346 & 3113 & 0.020000 & ex02 & 226 & 1547 & 0.070000 \\
\hline seba & 2 & 9 & 0.130000 & ex05 & 831 & 7747 & 0.090000 \\
\hline share $1 b$ & 107 & 243 & 0.040000 & ex06 & 824 & 7778 & 0.090000 \\
\hline share $2 b$ & 92 & 158 & 0.010000 & ex09 & 1821 & 18184 & 0.340000 \\
\hline shell & 487 & 1450 & 0.020000 & & & & \\
\hline
\end{tabular}




\subsection{Computational Results}

Table 2 shows the relative gain by each algorithm in all problems considering the five different times, i.e., the percentage of problems where the algorithm obtained the lowest value for the residual $\left\|b^{k}\right\|$, in the times $t 1$ up to $t 5$.

Table 2: Percentage of the relative gain by the algorithms on the problems in five different times

\begin{tabular}{lccccc}
\hline Algorithm & $\mathbf{t 1}$ & $\mathbf{t 2}$ & $\mathbf{t 3}$ & $\mathbf{t 4}$ & $\mathbf{t 5}$ \\
\hline Algorithm with $\mathrm{p}=2$ & $7.28 \%$ & $9.93 \%$ & $19.20 \%$ & $4.63 \%$ & $11.92 \%$ \\
Algorithm with $\mathrm{p}=4$ & $23.84 \%$ & $28.47 \%$ & $23.17 \%$ & $19.20 \%$ & $19.20 \%$ \\
Algorithm with $\mathrm{p}=10$ & $17.88 \%$ & $17.21 \%$ & $15.89 \%$ & $19.21 \%$ & $13.25 \%$ \\
Algorithm with $\mathrm{p}=20$ & $23.85 \%$ & $6.63 \%$ & $8.63 \%$ & $27.81 \%$ & $3.98 \%$ \\
Algorithm with $\mathrm{p}=40$ & $4.64 \%$ & $4.64 \%$ & $1.33 \%$ & $2.66 \%$ & $9.27 \%$ \\
Algorithm with $\mathrm{p}=100$ & $22.51 \%$ & $33.12 \%$ & $31.78 \%$ & $26.49 \%$ & $42.38 \%$ \\
\hline
\end{tabular}

According with Table 2, for the optimal adjustment algorithm for $p=100$ and $p=4$ coordinates, more problems obtain the lowest value of the residual in comparison with the OPAA $(p=2)$ on the five times. And for $p=4$, the performance was worse. Especially, the optimal adjustment algorithm for $p=100$ coordinates had better performance.

The performances of the algorithms using performance profile [6] was also analyzed. The distance of the residual $\left\|b^{k}\right\|$ to the origin was used to measure the performance. In these graphs, $\rho(1)$ represents the algorithm's total gain. The best performance algorithms are the ones above the others in graphics.

In Figure 6, the performance profile of the six algorithms with time $t 5$ are compared. This figure shows that the optimal adjustment algorithm for $p$ coordinates is more efficient than the OPAA $(p=2)$ for $p=100, p=10$ and $p=4$. However the algorithm loses in performance for $p=20$ and $p=40$. Table 2 shows the performance of the algorithms in the t5 column. In terms of robustness, for all values of $p$ the behaviour is the same.

Figures 9, 10, and 7 show the performance profiles among family algorithms for $p=100, p=40$, $p=20, p=10$, and $p=4$ and OPAA at the time $t 5$. The five figures show that the five family algorithms are more efficient and robust. The highest efficiency was achieved by the algorithm with $p=4$, which had $88 \%$ efficiency, followed by the algorithm with $p=10$, which had $84 \%$ efficiency, followed by the algorithm with $p=20$, which had a $74 \%$ efficiency, followed by the algorithm with $p=40$, which had a $60 \%$ efficiency and ending with the algorithm with $p=100$, which had a $57 \%$ efficiency. Thus, at time $t 5$, the family algorithms achieved higher efficiency when compared with OPAA. As for robustness, the five figures show that the curves from the five family algorithms are on top of the curve from the OPAA, thus demonstrating their strength. 


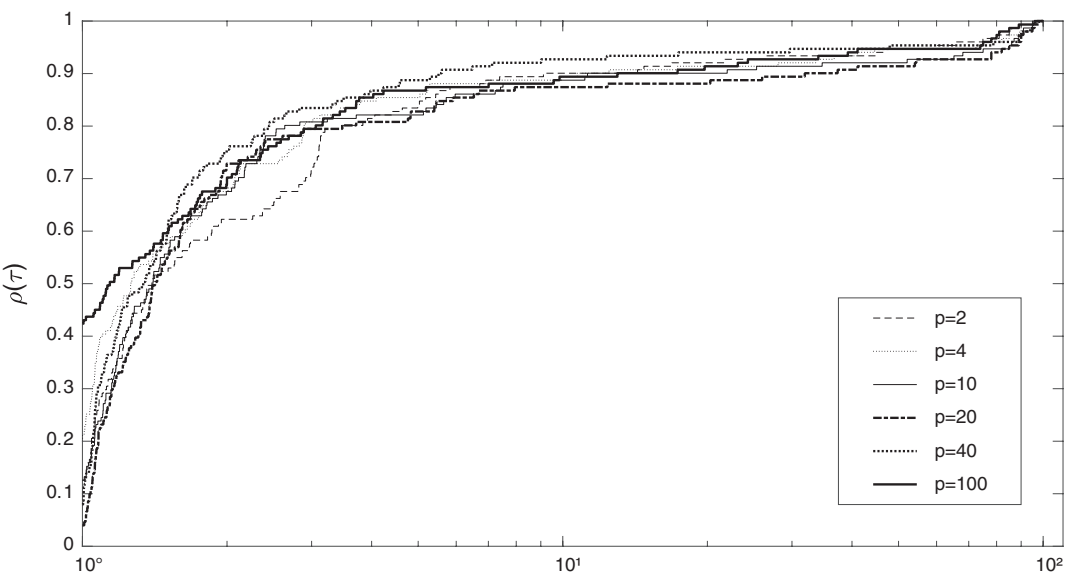

Figure 6: Performance profile of six algorithms in t5 time.

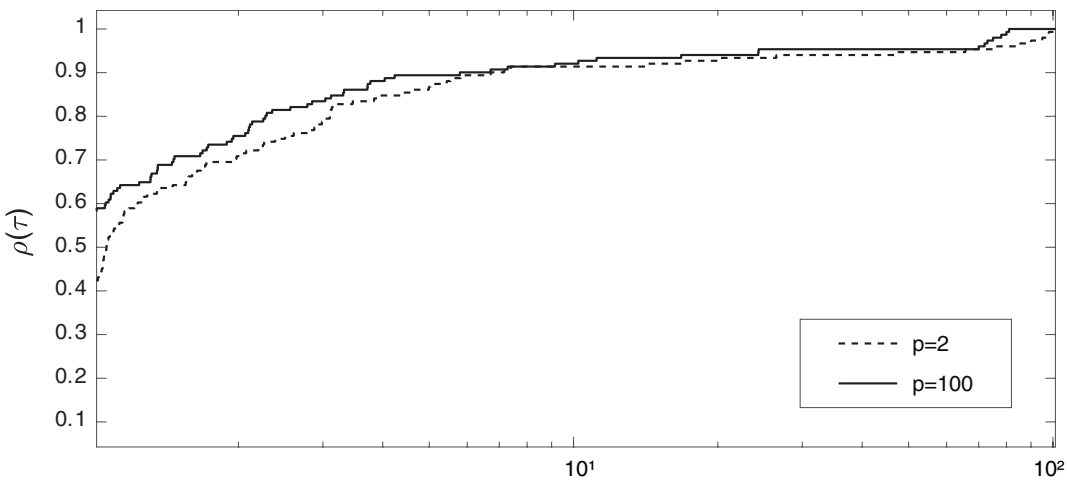

Figure 7: Performance profile of the algorithms with $p=2$ and $p=100$ in time $t 5$.

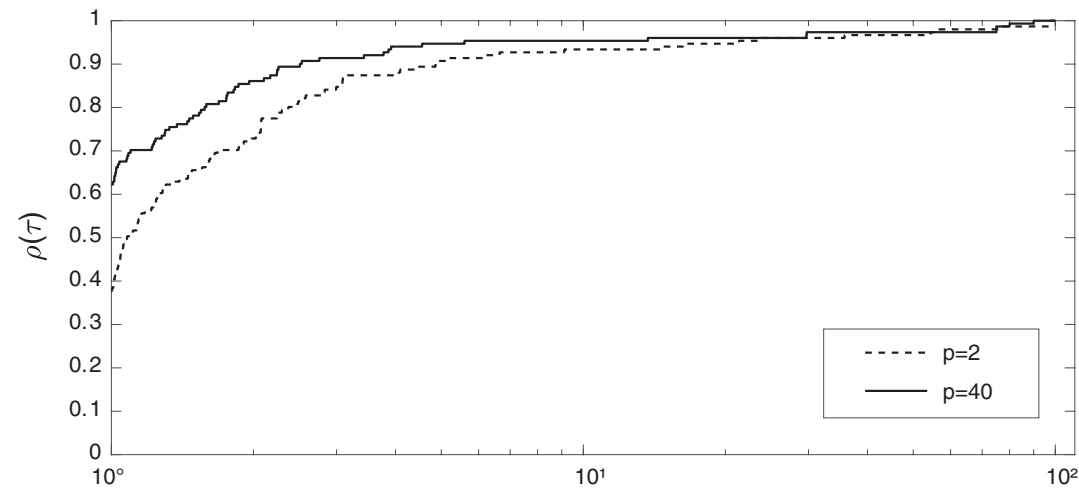

Figure 8: Performance profile of the algorithms with $p=2$ and $p=40$ in time $t 5$. 


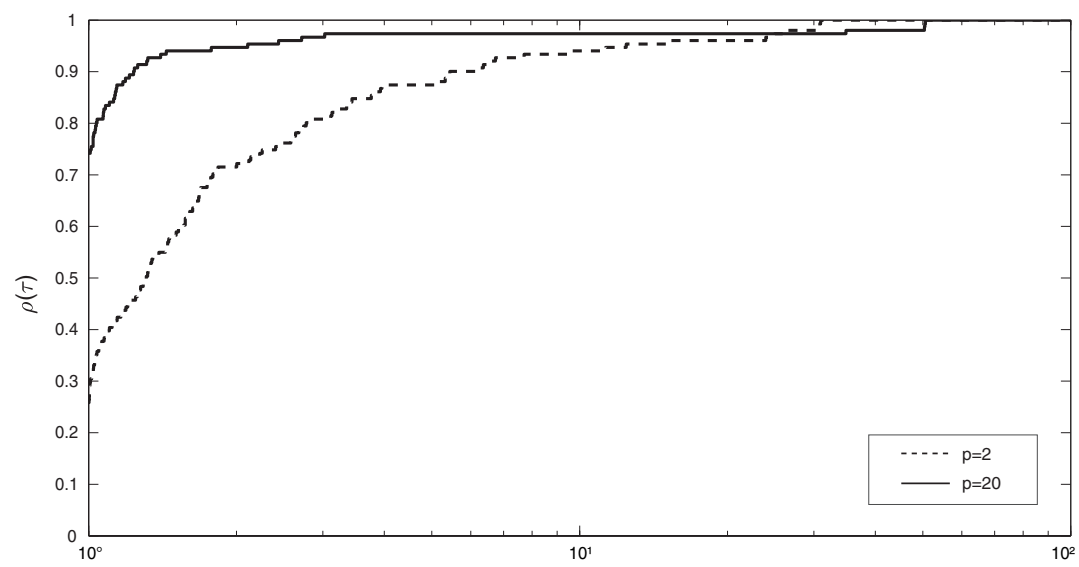

Figure 9: Performance profile of the algorithms with $p=2$ and $p=20$ in time $t 5$.

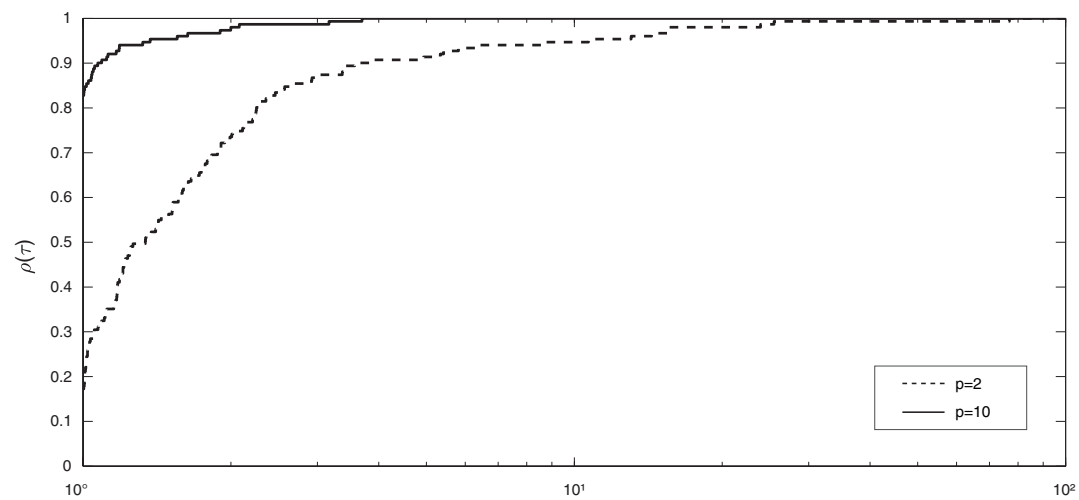

Figure 10: Performance profile of the algorithms with $p=2$ and $p=10$ in time $t 5$.

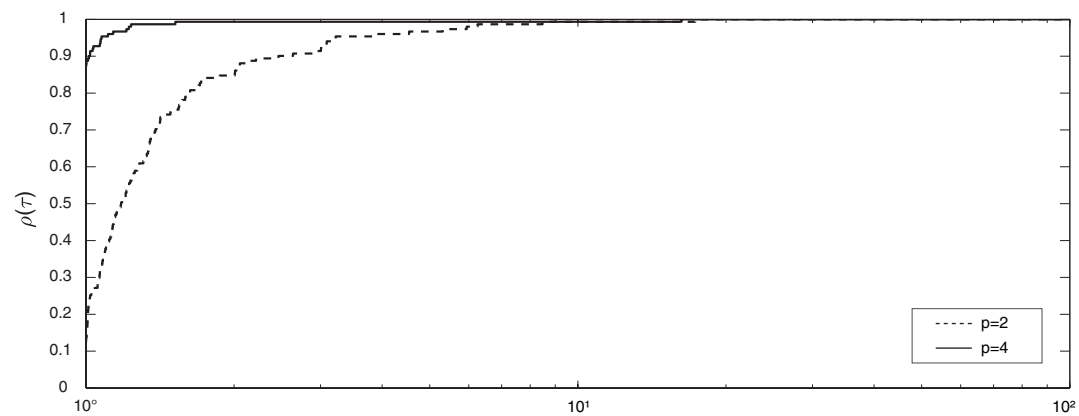

Figure 11: Performance profile of the algorithms with $p=2$ and $p=4$ in time $t 5$. 
Thus, the performance profiles show that the family of algorithms has good performance for moderate $p$ values.

The results obtained in the second experiment are presented in Table 3. The best-performing approach is given by the heuristic $p=\frac{n z(A)}{\sqrt{m n}}$. It achieves a smaller number of iterations in 52\% of the 151 problems tested, a lager number of iterations in $35 \%$ of the problems and the same number of iterations in $13 \%$ of them. In the Table 3 we present the value of $p$ and the number of iterations (it) for the approach in [8] (H) and the new heuristic (NH).

Table 3: Heuristic: $H \times N H$

\begin{tabular}{|c|c|c|c|c|c|c|c|c|c|}
\hline \multirow[b]{2}{*}{ Problem } & \multicolumn{2}{|l|}{$\mathrm{H}$} & \multicolumn{2}{|l|}{$\mathrm{NH}$} & \multirow[b]{2}{*}{ Problem } & \multicolumn{2}{|l|}{$\mathrm{H}$} & \multicolumn{2}{|l|}{$\mathrm{NH}$} \\
\hline & $\mathrm{p}$ & it & $\mathrm{p}$ & it & & $\mathrm{p}$ & it & $\mathrm{p}$ & it \\
\hline $25 \mathrm{fv} 47$ & 4 & 100 & 9 & 100 & ship08s & 4 & 100 & 6 & 100 \\
\hline 80bau3b & 8 & 47 & 3 & 17 & ship121 & 4 & 100 & 6 & 100 \\
\hline adlittle & 4 & 100 & 5 & 100 & ship12s & 4 & 100 & 6 & 100 \\
\hline afiro & 4 & 100 & 3 & 100 & sierra & 4 & 100 & 2 & 100 \\
\hline agg & 4 & 100 & 5 & 49 & stair & 4 & 100 & 9 & 100 \\
\hline agg2 & 4 & 6 & 8 & 63 & standata & 4 & 100 & 3 & 100 \\
\hline agg3 & 4 & 6 & 8 & 8 & standgub & 4 & 100 & 3 & 100 \\
\hline bandm & 4 & 100 & 7 & 100 & standmps & 4 & 100 & 4 & 100 \\
\hline beaconfd & 4 & 100 & 11 & 100 & stocfor1 & 4 & 100 & 4 & 100 \\
\hline blend & 4 & 100 & 6 & 100 & stocfor 2 & 4 & 100 & 4 & 100 \\
\hline bnl1 & 4 & 100 & 6 & 100 & stocfor3 & 20 & 100 & 4 & 100 \\
\hline bnl2 & 4 & 100 & 6 & 100 & truss & 4 & 100 & 10 & 100 \\
\hline boeing1 & 4 & 100 & 5 & 100 & tuff & 4 & 46 & 11 & 100 \\
\hline boeing2 & 4 & 100 & 4 & 100 & wood1p & 4 & 100 & 83 & 3 \\
\hline bore3d & 4 & 100 & 5 & 100 & woodw & 4 & 100 & 11 & 100 \\
\hline brandy & 4 & 100 & 11 & 100 & pds-10 & 20 & 100 & 3 & 100 \\
\hline capri & 4 & 100 & 4 & 100 & pds-20 & 20 & 100 & 2 & 100 \\
\hline cycle & 4 & 100 & 8 & 100 & scagr25 & 4 & 100 & 4 & 100 \\
\hline czprob & 4 & 100 & 5 & 100 & gfrdpnc & 4 & 100 & 3 & 100 \\
\hline $\mathrm{d} 2 \mathrm{q} 06 \mathrm{c}$ & 4 & 100 & 10 & 100 & pilotja & 4 & 100 & 8 & 100 \\
\hline d6cube & 4 & 100 & 24 & 100 & pilotwe & 4 & 82 & 6 & 100 \\
\hline degen 2 & 4 & 100 & 8 & 100 & vtpbase & 4 & 63 & 3 & 100 \\
\hline degen 3 & 4 & 100 & 13 & 100 & cre-a & 4 & 100 & 4 & 100 \\
\hline dfl001 & 8 & 100 & 5 & 3 & cre-b & 20 & 100 & 9 & 100 \\
\hline e226 & 4 & 100 & 9 & 100 & cre-c & 4 & 100 & 4 & 100 \\
\hline etamacro & 4 & 13 & 4 & 13 & cre-d & 20 & 100 & 9 & 100 \\
\hline fffff800 & 4 & 100 & 11 & 100 & ken-07 & 4 & 100 & 2 & 100 \\
\hline finnis & 4 & 100 & 4 & 100 & ken11 & 20 & 100 & 2 & 100 \\
\hline fit1d & 4 & 33 & 10 & 53 & ken13 & 20 & 100 & 2 & 100 \\
\hline fit $1 p$ & 4 & 100 & 7 & 100 & ken18 & 20 & 100 & 2 & 100 \\
\hline fit $2 d$ & 8 & 5 & 9 & 5 & osa-07 & 20 & 100 & 18 & 45 \\
\hline fit $2 p$ & 8 & 100 & 4 & 100 & osa-14 & 20 & 65 & 18 & 71 \\
\hline forplan & 4 & 6 & 18 & 96 & osa-30 & 20 & 73 & 18 & 67 \\
\hline
\end{tabular}


Table 3 (Continued from previous page)

\begin{tabular}{|c|c|c|c|c|c|c|c|c|c|}
\hline \multirow[b]{2}{*}{ Problem } & \multicolumn{2}{|l|}{$\mathrm{H}$} & \multicolumn{2}{|l|}{$\mathrm{NH}$} & \multirow[b]{2}{*}{ Problem } & \multirow{2}{*}{$\begin{array}{c}\mathrm{H} \\
\mathrm{p}\end{array}$} & \multicolumn{3}{|c|}{$\mathrm{NH}$} \\
\hline & $\mathrm{p}$ & it & $\mathrm{p}$ & it & & & it & $\mathrm{p}$ & it \\
\hline ganges & 4 & 2 & 4 & 2 & osa- 60 & 20 & 100 & 2 & 9 \\
\hline greenbea & 4 & 54 & 8 & 40 & pds-02 & 4 & 100 & 3 & 100 \\
\hline greenbeb & 4 & 100 & 8 & 68 & pds-06 & 20 & 100 & 3 & 100 \\
\hline grow15 & 4 & 100 & 6 & 100 & $\mathrm{BL}$ & 8 & 100 & 4 & 100 \\
\hline grow22 & 4 & 100 & 6 & 100 & BL2 & 8 & 100 & 4 & 100 \\
\hline grow7 & 4 & 100 & 6 & 100 & $\mathrm{CO} 5$ & 8 & 100 & 7 & 100 \\
\hline israel & 4 & 100 & 11 & 100 & $\mathrm{CO} 9$ & 20 & 100 & 7 & 100 \\
\hline kb2 & 4 & 100 & 5 & 100 & CQ9 & 20 & 100 & 7 & 100 \\
\hline lotfi & 4 & 55 & 5 & 100 & GE & 20 & 6 & 4 & 6 \\
\hline maros & 4 & 6 & 7 & 72 & NL & 20 & 100 & 4 & 100 \\
\hline maros-r7 & 4 & 28 & 26 & 100 & a1 & 4 & 100 & 3 & 100 \\
\hline modszk1 & 4 & 25 & 3 & 34 & fort45 & 4 & 62 & 3 & 84 \\
\hline nesm & 4 & 100 & 5 & 100 & fort46 & 4 & 100 & 3 & 100 \\
\hline perold & 4 & 100 & 6 & 100 & fort47 & 4 & 83 & 3 & 100 \\
\hline pilot & 4 & 100 & 12 & 54 & fort48 & 4 & 92 & 3 & 100 \\
\hline pilot4 & 4 & 100 & 8 & 100 & fort49 & 4 & 34 & 3 & 54 \\
\hline pilot87 & 4 & 100 & 14 & 100 & fort51 & 4 & 42 & 4 & 42 \\
\hline pilotnov & 4 & 100 & 8 & 52 & fort52 & 4 & 100 & 3 & 100 \\
\hline recipe & 4 & 100 & 4 & 100 & fort53 & 4 & 100 & 3 & 100 \\
\hline sc105 & 4 & 100 & 3 & 100 & fort54 & 4 & 36 & 3 & 100 \\
\hline $\operatorname{sc} 205$ & 4 & 100 & 3 & 100 & fort55 & 4 & 34 & 3 & 100 \\
\hline $\mathrm{sc} 50 \mathrm{a}$ & 4 & 100 & 3 & 100 & fort56 & 4 & 100 & 3 & 100 \\
\hline $\mathrm{sc} 50 \mathrm{~b}$ & 4 & 100 & 3 & 100 & fort57 & 4 & 100 & 3 & 100 \\
\hline scagr25 & 4 & 100 & 4 & 100 & fort58 & 4 & 100 & 3 & 100 \\
\hline scagr7 & 4 & 100 & 3 & 100 & fort59 & 4 & 100 & 3 & 100 \\
\hline scfxm1 & 4 & 100 & 6 & 100 & fort60 & 4 & 100 & 3 & 100 \\
\hline scfxm2 & 4 & 100 & 6 & 100 & fort61 & 4 & 100 & 3 & 100 \\
\hline scfxm3 & 4 & 100 & 6 & 100 & $\mathrm{x} 1$ & 4 & 4 & 3 & 6 \\
\hline scorpion & 4 & 100 & 4 & 100 & $\mathrm{x} 2$ & 4 & 100 & 3 & 76 \\
\hline scrs8 & 4 & 100 & 5 & 100 & pata01 & 4 & 100 & 7 & 100 \\
\hline $\operatorname{scsd} 1$ & 4 & 100 & 10 & 100 & pata02 & 4 & 100 & 7 & 100 \\
\hline $\operatorname{scsd} 6$ & 4 & 100 & 10 & 100 & patb01 & 4 & 100 & 4 & 100 \\
\hline $\operatorname{scsd} 8$ & 4 & 100 & 9 & 100 & patb02 & 4 & 100 & 4 & 100 \\
\hline sctap1 & 4 & 100 & 5 & 100 & vschna02 & 4 & 100 & 7 & 100 \\
\hline sctap2 & 4 & 100 & 5 & 100 & vschnb01 & 4 & 100 & 4 & 100 \\
\hline sctap3 & 4 & 100 & 5 & 100 & vschnb02 & 4 & 100 & 4 & 100 \\
\hline seba & 4 & 100 & 4 & 100 & willett & 4 & 100 & 8 & 100 \\
\hline share $1 b$ & 4 & 88 & 7 & 100 & ex01 & 4 & 100 & 5 & 100 \\
\hline share2b & 4 & 100 & 7 & 100 & ex02 & 4 & 100 & 5 & 100 \\
\hline shell & 4 & 68 & 3 & 100 & ex05 & 4 & 100 & 5 & 100 \\
\hline ship041 & 4 & 100 & 6 & 100 & ex06 & 4 & 100 & 5 & 100 \\
\hline ship04s & 4 & 100 & 6 & 100 & ex09 & 20 & 100 & 5 & 100 \\
\hline ship081 & 4 & 100 & 6 & 100 & & & & & \\
\hline
\end{tabular}




\section{CONCLUSIONS}

The family of simple algorithms arose from the generalization of the OPAA. The major advantage of this family of algorithms is its simplicity and fast initial convergence. This paper presents a comparison between a family of simple algorithms for linear programming and the OPAA. Besides, it is proved that the algorithm with $p=1$ is equivalent to von Neumann's algorithm. Finally, sufficient conditions show that the family of algorithms has better performance than von Neumann's algorithm.

The first experiment is performed in a similar framework as reported by in Gonçalves, Storer and Gondzio in [11] and the second experiment is performed in a similar framework as reported in [9]. In the first experiment the computational results show the superiority of the family of algorithms for moderate $p$ values, in comparison with OPAA. Performance profile graphs indicate that the family of algorithms has significantly more efficiency and robustness. In the second experiment the new heuristic $p=\frac{n z(A)}{\sqrt{m n}}$ achieves better results than the approach given in [9].

Despite the improvements with respect to OPAA, the family of algorithms is not practical for solving linear programming problems up to a solution. However, it can be useful in some instances, such as, improving the starting point for interior point methods as in [8], or to work in combination with interior point methods using its initial fast convergence rate as reported in [9]. Nevertheless, future researches are needed to measure the impact that the family of algorithms can have in this direction.

\section{ACKNOWLEDGEMENT}

This work was partially funded by the Foundation for the Support of Research of the State of São Paulo (FAPESP) and Brazilian Council for the Development of Science and Technology (CNPq). The authors are grateful to the referees for their valuable suggestions which improved this article.

RESUMO. Este artigo apresenta uma comparação entre uma família de algoritmos simples para programação linear e o algoritmo de ajustamento pelo par ótimo. O algoritmo de ajustamento pelo par ótimo foi desenvolvido para melhorar a convergência do algoritmo de von Neumann que é um algoritmo muito interressante por causa de sua simplicidade. Porém não é muito prático resolver problemas de programação linear até a otimalidade com ele, visto que sua convergência ainda é muito lenta. A família de algoritmos simples surgiu da generalização do algoritmo de ajustamento pelo para ótimo, incluindo um parâmetro sobre o número de colunas escolhidas, em vez de manter fixa duas. Esta generalização preserva a simplicidade dos algoritmos e suas boas qualidades. Apresentamos experimentos numéricos sobre um conjunto de problemas de programação linear que mostram melhorias significativas em relação ao algoritmo de ajustamento pelo par ótimo.

Palavras-chave: Programação Linear, Algoritmo de von Neumann, Algoritmos Simples. 


\section{REFERENCES}

[1] A. Beck \& M. Teboulle. A Conditional Gradient Method with Linear Rate of Convergence for Solving Convex Linear Systems. Mathematical Methods of Operations Research, (2004).

[2] W. Carolan, J. Hill, J. Kennington, S. Niemi \& S. Wichmann. An empirical evaluation of the KORBX algorithms for military airlift applications. Oper. Res, 38 (1990), 240-248.

[3] N. collection LP test sets. NETLIB LP repository. Online at http://www.netlib.org/lp/data.

[4] G.B. Dantzig. Converting a converging algorithm into a polynomially bounded algorithm. Technical report, Stanford University (1991).

[5] G.B. Dantzig. An $\varepsilon$-precise feasible solution to a linear program with a convexity constraint in $\frac{1}{\varepsilon^{2}}$ iterations independent of problem size. Technical report, Stanford University (1992).

[6] E. Dolan \& J. More. Benchmarking optimization software with performance profiles. Math. Program, (2002), 91:201-213.

[7] M. Epelman \& R.M. Freund. Condition number complexity of an elementary algorithm for computing a reliable solution of a conic linear system. Mathematical Programing, 88 (2000), 451-485.

[8] C.T.L.S. Ghidini, A.R.L. Oliveira \& J. Silva. Optimal Adjustment Algorithm for p Coordinates and the Starting Point in Interior Point Methods. American Journal of Operations Research, (2011).

[9] C.T.L.S. Ghidini, A.R.L. Oliveira, J. Silva \& M. Velazco. Combining a Hybrid Preconditioner and a Optimal Adjustment Algorithm to Accelerate the Convergence of Interior Point Methods. Linear Algebra and its Applications, 436 (2012), 1-18.

[10] J.P.M. Gonçalves. "A Family of Linear Programming Algorithms Based on the Von Neumann Algorithm”. Ph.D. thesis, Lehigh University, Bethlehem (2004).

[11] J.P.M. Gonçalves, R.H. Storer \& J. Gondzio. A Family of Linear Programming Algorithms Based on an Algorithm by von Neumann. Optimization Methods and Software, (2009).

[12] J. Silva. "Uma Família de Algoritmos para Programação Linear Baseada no Algoritmo de Von Neumann”. Ph.D. thesis, IMECC - UNICAMP, Campinas SP (2009). In portuguese. 